\title{
A System Architecture for Online Data Interpretation and Reduction in Fluorescence Microscopy
}

\author{
Thorsten Röder ${ }^{a}$ and Matthias Geisbauer ${ }^{b}$ and Yang Chen ${ }^{a}$ and Alois Knoll ${ }^{a}$ and Rainer $\mathrm{Uhl}^{b}$ \\ ${ }^{a}$ Technische Universität München, \\ Boltzmannstr. 3, 85748 Garching bei München, Germany; \\ ${ }^{b}$ BioImaging Zentrum of Ludwig-Maximilians-Universität, \\ Großhaderner Str. 2-4, 82152 Martinsried, Germany
}

\begin{abstract}
In this paper we present a high-throughput sample screening system that enables real-time data analysis and reduction for live cell analysis using fluorescence microscopy. We propose a novel system architecture capable of analyzing a large amount of samples during the experiment and thus greatly minimizing the post-analysis phase that is the common practice today. By utilizing data reduction algorithms, relevant information of the target cells is extracted from the online collected data stream, and then used to adjust the experiment parameters in real-time, allowing the system to dynamically react on changing sample properties and to control the microscope setup accordingly. The proposed system consists of an integrated DSP-FPGA hybrid solution to ensure the required real-time constraints, to execute efficiently the underlying computer vision algorithms and to close the perception-action loop. We demonstrate our approach by addressing the selective imaging of cells with a particular combination of markers. With this novel closed-loop system the amount of superfluous collected data is minimized, while at the same time the information entropy increases.
\end{abstract}

Keywords: fluorescence microscopy, high-throughput, sample screening, live cell analysis, DSP, FPGA, computer vision, closed-loop system

\section{MOTIVATION}

Fluorescence microscopy falls into the category of light microscopy techniques, especially exploiting the excitation properties of special light sensitive molecules, called fluorophores. Fluorophores have the property to be excited by certain light wavelength causing them to re-emit light of light of a longer wavelength. ${ }^{1}$ In the last several years many other techniques, besides the standard transillumination microscopy approach, have been developed, such as fluorescence resonance energy transfer (FRET), fluorescence recovery after photobleaching (FRAP), total internal reflection fluorescence (TIRF) or laser scanning. Nowadays a variety of different fluorophores, like dyes, such as DAPI, or fluorescent proteins, such as green fluorescent protein (GFP) are known and used on a daily basis for all kinds of experiments, especially in cell and molecular biology. ${ }^{1-3}$ With these advances, the cell biology and the corresponding fluorescence microscopy techniques more and more find their way into the drug discovery domain..$^{4-6}$ For this domain many live cell assays for high-throughput screening have been developed and also successfully used in combination with fluorescence microscopy, which in general has become a powerful tool for the live cell assay analysis. ${ }^{7}$

As the screening of more than 100.000 samples per day is the current daily standard, limited regular life science microscopes can no longer be used and highly integrated as well as automated high-throughput screening microscope platforms have to be employed. ${ }^{12}$ When using fluorescence microscopy in high-throughput screening scenarios, the huge amount of collected data at a high frequency is the major drawback that limit its applicability. Here the amount of collected data is likely to reach the terabyte dimension during a single day of a single experiment and an information management software is required. ${ }^{13}$ Therefore without applying sophisticated additional online data processing steps, the handling and storing of data becomes difficult in both dimensions: time and space, which in turn limits the data and biological sample throughput.

E-mail: roeder@in.tum.de, geisbauer@biz.uni-muenchen.de, yang.chen@in.tum.de, knoll@in.tum.de, uhl@biz.unimuenchen.de 
The past trends of shifting the analysis of data towards a separate post-processing phase are changing ${ }^{8-10}$ to more scalable and automated online data processing systems, which minimize or even avoid the amount of intermediately stored data. This also means that traditional strategies of retaining raw high-resolution images have to be revised and data interpretation as well as data reduction techniques become key aspects for scalability with respect to high-throughput screening applications, which today require the need of high-capacity and fast storage systems. Besides the storage issues, the increasing computational effort that is required to process and analyze this data also constitutes a performance bottleneck in todays microscopy applications. ${ }^{11}$ These issues become even more important when it comes to large-scale screening experiments that typically involve large multi-well dishes for assay investigation (maybe also using banks of microscopes) for which high-throughput is the most critical key aspect.

In this paper we propose a novel modular and adaptive microscopy architecture that addresses the mentioned issues of high-throughput systems for live cell analysis by employing intelligent online data processing methodologies. The execution of interleaved concurrent data acquisition and data processing steps during the execution of an assay renders possible the shortening of traditional post-analysis phases. Additionally by employing online processing methodologies, the autonomous interference during the experiment, for example the real-time adaptation of various assay properties, become possible.

This paper is organized as follows: section 2 describes the underlying system architecture and used hardware setup, section 3 handles the basic visual processing steps, section 4 gives some results regarding time and space constraints and section 5 concludes the proposed approach.

\section{SYSTEM ARCHITECTURE AND HARDWARE SETUP}

Usually microscopy systems involve a state-of-the art microscope that is connected to a stand-alone PC, but there exist also some industrial systems are more advanced and make use of computing clusters and dedicated storage systems in order to handle the huge data amount. Assays typically follow a pre-defined and a-priori fixed process and the controlled adaption according to changes of the experimental environment is based on a predefined static process plan and thus hard to achieve. For current systems the dynamic handling and adaption of environmental parameters according to vision-based target functions and this way closing the perception-action loop is in its infancy.

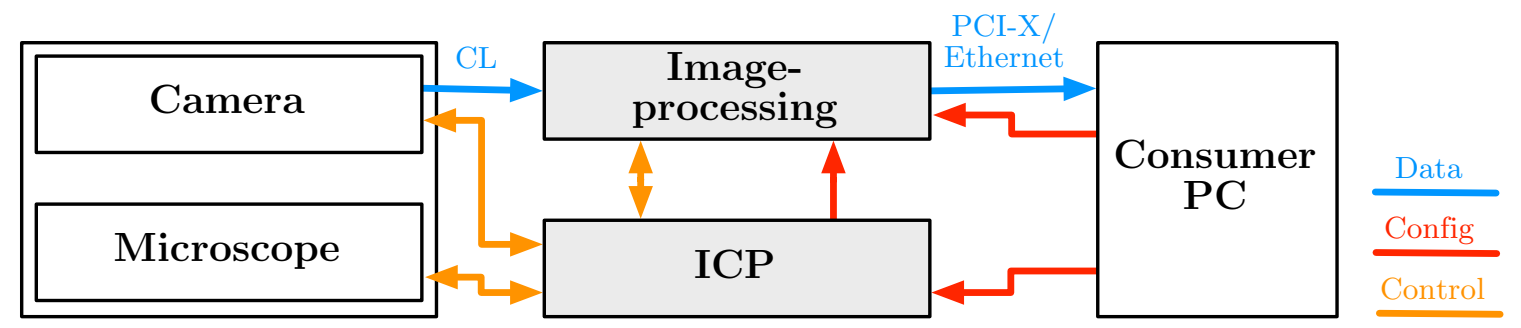

Figure 1. Hardware architecture consisting of a microscope, an integrated control platform (ICP), embedded image preprocessing unit and a consumer PC for assay configuration, monitoring, online reconfiguration and statistical evaluation. The main bandwidth is required for the camera connection, which uses a CameraLink interface (CL), and the connection to the PC, which is either a PCI-X or a gigabit Ethernet interface. The current setup communicates over the PCI-X bus. For scalability, especially when using multiple microscopes in parallel the Ethernet standard was chosen as a more scalable interface.

The proposed architecture enhances the state-of-the-art fluorescence microscopy solutions by adding two additional compute and control components as depicted in figure 1. The compute component is dedicated to the visual processing of the incoming raw image feed of the camera, being able to operate on lower pixel-level as well as to provide information about cell properties based on higher level visual feature extraction. The ICP itself is able to operate the microscope in real-time closed loop control. Both components are implemented as self-contained embedded solutions in order to fulfill real-time and data bandwidth constraints as described below. 
Our demonstration system integrates a MORE microscope (TILL Photonics GmbH*, Martinsried, Germany) depicted in figure 2, which can perform fully automated microscopy assays. Novel LED and laser illumination techniques combined with an extremely stable motorized X-Y-stage, ultra fast dichroic and objective changers and a high precision voice-coil-based focus drive with integrated focus clamp turn the microscope into an ideally suited system for automated high-throughput microscopy, including all major microscopy techniques like transillumination, FRET, FRAP, TIRF and laser scanning.

The sophisticated and fully integrated DSP-FPGA based control architecture, which is an enhancement of the Integration Control Unit (ICU) ${ }^{14}$ (figure 2), allows hardware real-time microscope control and synchronization of all integrated components such as X-Y-stage, focus drive and scanners on a time base of $10 \mu s$.

For controlling functions that do require a more precise timing, the synchronization is done on a $1 \mu s$ base: e.g. in order to avoid divergency in experiment conditions like exposure time triggers or image acquisition triggers. Unlike the former modular microscope control system, the new integrated control platform (ICP) services all microscope intern interfaces directly on a full scale: the control of the X-Y-stage, the focus drive and the image acquisition, which results in an fully independently working screening system. Besides the experiment control, the ICP also provides a standard Ethernet PC interface and thus makes the system ideally suited for parallel usage of more than one microscope per acquisition PC.

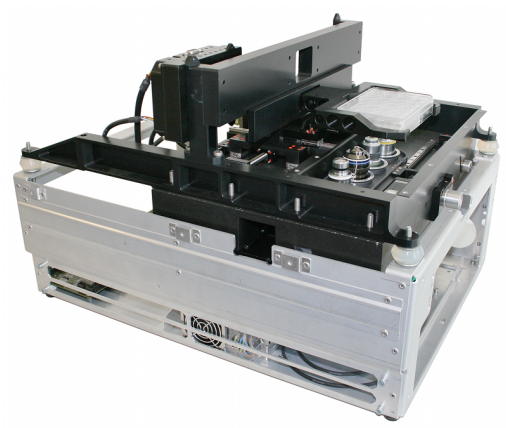

(a) MORE microscope (open frame)

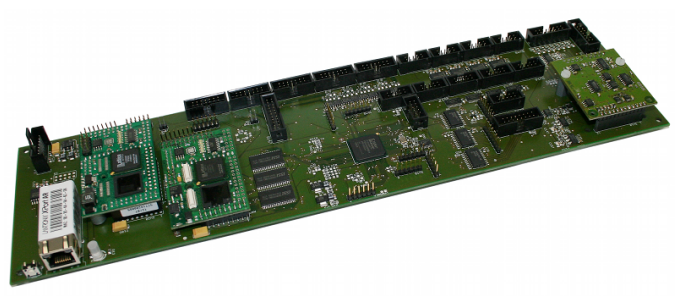

(b) Integrated Control Platform (ICP) board

Figure 2. The utilized microscope and the embedded ICP control board.

The ICP provides operational sequence features like loops or if-else conditions, which are usually just known from higher programming language and enables the application of complex protocols and then run in real-time, giving the system the ability of acquiring sets of statistically relevant data. The combination of a pco.1600 CCD camera $\left(\mathrm{PCO}^{\dagger}\right.$, Kelheim, Germany) with a programmable image data processing frame grabber ProcStarIII (Gidel ${ }^{\ddagger}$, Or Akiva, Israel), both connected via a CameraLink interface, enables acquisition and online data interpretation. The utilized camera has a spatial sensor resolution of $1600 \times 1200$ pixel, each providing a dynamic range of 14 bit at a upper framerate of $30 \mathrm{fps}$.

Coupling the visual data pre-processing module with the real-time environment control gives us the possibility of adapting the experiment in real-time and thus provides a powerful feedback loop for time minimizing and content maximizing optimization based on a image content analysis.

Our proposed system architecture allows the online visual processing and real-time active control. In this paper we show a selective data reduction technique based on extracted visual features. The system takes an overview picture using a objective with a lower magnification and extracts the required visual properties in order to decide whether or not the perceived field of view contains valuable information that should be considered in more detail. By introducing this additional decision step prior to spending a reasonable amount of time analyzing uninteresting detailed information, the amount of data as well as the time for analysis and transmission can be minimized.

\footnotetext{
*http://www.till-photonics.com

${ }^{\dagger}$ http: //www.pco.de

${ }^{\ddagger}$ http://ww. gidel.com
} 
High-throughput can be seen in two different views: a) related to the bandwidth of the imaging data or b) e.g. the bandwidth of the classified cell information. The upper input data rate of the presented system is defined by the image size and the upper framerate. Using a camera with $1600 \times 1200 \times 14$ bit $\times 30 f p s$ yields an estimated incoming data rate of $96.13 \mathrm{MB}$ per second.

\section{VISUAL PROCESSING}

Visual processing represents the fundamental backbone of the microscopy system (for adaptive control, data interpretation and data reduction). Although sophisticated assay-specific visual processing algorithms are available today, most of them are not prepared for the use in high-throughput imaging, ${ }^{10}$ because they have to provide repeatable, reliable and quantitative results in an automated way in order to be suitable for statistical evaluation. This especially turns out to be complex when dealing with live biological cell analysis as processing algorithms have to be prepared for irregularities, varying cell properties and dynamic visual shape and appearance, signal to noise ratio, inherent temporal physical constraints, like for example the photobleaching effect. In order to automatically react to a dynamical environment, the use of static pre-defined rules as they are typically implemented in state-of-the-art experiment profiles is limited.

In order to cope with the high dimensional dynamics in a high-throughput screening system we follow a suggestion of using dedicated visual processing modules that are linked in a fixed processing sequence. ${ }^{10}$ Table 1 gives an overview of the processing modules. The concrete implementation for each of the module varies dependent of the concrete assay requirements. Although the concrete implementation might change for a class of experiments, the overall processing order of the modules remains the same. Some assays do not require to execute all modules due to their configuration. Thus the visual processing pipeline can on the one hand be stopped at any stage and on the other hand can have omitted modules.

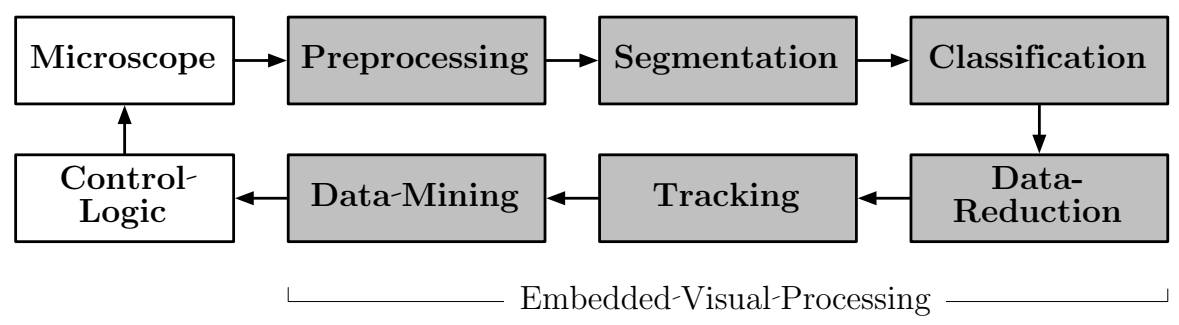

Figure 3. System components and visual processing modules for realizing the closed loop control: a microscope (imaging device, secondary sensors and actuators), an experiment logic control and several interlinked visual processing and interpretation submodules.

Figure 3 depicts the implemented closed loop control and shows the several steps that are considered in our visual processing system. It is important to mention that during a single iteration (frame) every module can access the intermediate extracted or computed results of any previously executed processing module. After capturing the camera frame, the preprocessing module is responsible for a pixel-level target-model-free image pre-processing. Here for example a typical flat-field and dark frame correction for noise compensation and shading correction ${ }^{15,16}$ can be applied:

$$
\mathcal{I}_{n}(x, y)=K \frac{\mathcal{I}_{c}(x, y)-\mathcal{I}_{d}(x, y)}{\mathcal{I}_{f}(x, y)-\mathcal{I}_{d}(x, y)}
$$

where $K$ is a setup dependent user-defined scaling constant, $\mathcal{I}_{n}$ is the normalized image, $\mathcal{I}_{c}$ is the raw input image captured by the CCD camera, $\mathcal{I}_{d}$ is the average dark frame of the camera captured with closed shutter and $\mathcal{I}_{f}$ is the average flat-field image in order to achieve spatial-invariant lightning conditions. Additional cell-model-free lower level pre-processing can also take place here. For example, if the image to be analyzed would consist of several individual, but spatial-correlated, images, they could also be stitched together by this pre-processing module. 
Table 1. Overview of the visual processing modules and a brief description of their main task within the processing pipeline.

\begin{tabular}{lcrr}
\hline Processing step & Module & Description & Output \\
\hline 1. & preprocessing & processing of the raw input image & normalized image \\
2. & segmentation & cell detection & regions of special interest \\
3. & classification & cell recognition & cell classification \\
4. & data reduction & data reduction & lossy or lossless compressed data \\
5. & tracking & sequential estimation & object associations \\
6. & data mining & property estimation and aggregation & statistical features \\
\hline
\end{tabular}

The segmentation module depends on the normalized image $\mathcal{I}_{n}$ and extracts information about regions of interest. It is meant to cancel out the data that is by-definition not a object of interest. Here static or adaptive thresholding techniques or also local optimization based segmentation methods can be employed. Our evaluation setup performs a static intensity based thresholding decision algorithm. For this intensity cut-off we are assuming flat objects that do have a suitable continuous intensity profile and neglecting cases for which the aperture problem arises:

$$
\mathcal{I}_{\tau}(x, y)= \begin{cases}1 & \text { if } \mathcal{I}_{n}(x, y) \geq \tau \\ 0 & \text { else }\end{cases}
$$

where $\mathcal{I}$ represents a grayscale input image, $\mathcal{I}_{\tau}$ the respective thresholded binary image and $\tau$ the scalar threshold value. This technique is especially useful in fluorescence microscopy images, since in this application case the signal to noise ratio is beneficial by the actively emitting fluorophores. $\tau$ can either be chosen empirically or in most cases by using the mean image intensity:

$$
\tau=\frac{1}{A} \sum_{x, y} \mathcal{I}_{n}(x, y)
$$

where $A$ corresponds to the total image area measured in pixels by using $A=\mathcal{I}_{\text {width }} \times \mathcal{I}_{\text {height }}$. This computation depends on the visible cell culture density and is thus only applicable for lower culture densities with respect to the background intensity contribution.

The classification module takes the output information of the segmentation module, which currently consists of a binary image $\mathcal{I}_{\tau}$. We restrict ourselves to a simple classification scheme and interpret the two modes of the binary image, one as foreground (cell) and the other one as background (non-cell). More sophisticated classification algorithms may be integrated within this module, for example by using $\mathrm{Hu}^{17}$ or Zernike $\mathrm{Z}^{18}$ invariant moments, template matching, ${ }^{19}$ machine learning techniques. The classification module as well as the previously introduced segmentation module can access previous results of already processed frames (e.g. from the tracking module) in order to predict object positions and to assist the segmentation and classification procedure for the current frame.

For our experiments we compute the mean cell intensity and use this result as a decision criterion whether the current field of view contains enough valuable information that is worth for further, more time consuming, processing. This naive assumption depends on the cell properties that should be analyzed. For example: for detecting dead cells the classification needs to be adapted and has also to include suitable features. The average cell intensity can be estimated by computing:

$$
\alpha=\frac{\sum_{x, y} \mathcal{I}_{n}(x, y) \mathcal{I}_{\tau}(x, y)}{\sum_{x, y} \mathcal{I}_{\tau}(x, y)}
$$

where $\alpha$ represents the average cell density, $\mathcal{I}_{n}$ the normalized image of the preprocessing module and $\mathcal{I}_{\tau}$ the thresholded binary image of the segmentation module. Another classification criterion based on the usage of $\mathcal{I}_{\tau}$ would consist of the area portion covered by cells. The percentage of the image that is covered by the segmented foreground is given by:

$$
\alpha=\frac{1}{A} \sum_{x, y} \mathcal{I}_{\tau}(x, y)
$$


A further applicable acceptance criterion can be the average cell size:

$$
\alpha=\frac{1}{N} \sum_{x, y} \mathcal{I}_{\tau}(x, y)
$$

where $N$ represents the number of cells found, which equals to the number of distinct regions.

For certain assay conditions computing the average image intensity and using this as an acceptance criterion might already be sufficient:

$$
\alpha=\frac{1}{A} \sum_{x, y} \mathcal{I}_{n}(x, y)
$$

If $\alpha \geq \beta$ then we accept and spent more time to investigate $m$ detailed images, else we do not even capture these $m$ extra images. For demonstration purpose $m$ equals to 4 in this paper. The acceptance threshold $\beta$ depends on the experiment conditions and on the hardware setup. In order to cope with the intensity dynamics $\beta$ should get adapted over time. The acceptance criterion decides about the following steps. If the criterion evaluates to false, then the system believes that there is no interesting information to capture in a more detailed step. If the criterion evaluates to true, then the system knows that it should get more detailed information about the current field of view and it is worth investing more time to investigate it and to perform more time-intensive evaluations at the current position.

The data reduction module mainly relies on the output of the classification module as shown in figure 3 , but can also use all intermediate information collected or computed in the previous processing modules. Depending on the data reduction strategy, which also relies on the microscopy experiment in general, again the goal is to reduce as much information as possible. Especially interesting is the combination of data reduction techniques, which do not have any further knowledge about the cell domain, with algorithms that do have more information about the experimental setup itself. Here for example an additional data reduction could be achieved, if fewer bits per pixel can be used to encode the information:

$$
\tilde{\mathcal{I}}_{n, v_{\min }}(x, y)=\mathcal{I}_{n}(x, y)-v_{\min }
$$

where $v_{\max }=\max \mathcal{I}_{n}(x, y)$ is the maximum and $v_{\min }=\min \mathcal{I}_{n}(x, y)$ the minimum intensity value of $\mathcal{I}_{n} . \tilde{\mathcal{I}}_{n, v_{\min }}$ is the domain range shifted image from $v \in\left[v_{\min }, v_{\max }\right]$ to $v \in\left[0, v_{\max }-v_{\min }\right]$. The number of bits for for a lossless range encoding is given by $b=\left\lceil\log _{2}\left(\left\lceil v_{\max }\right\rceil-\left\lfloor v_{\min }\right\rfloor\right)\right\rceil$, consequently the minimum number of required bytes are determined by $\left\lceil\frac{1}{8} b\right\rceil$. The conversion of the image should only be done if the actual dynamic range is not fully used and the domain range fits into a lower bit requirement.

The tracking module is responsible to solve temporal data associations of multiple targets by applying sequential estimators like Bayesian filters. This makes it possible to restrict the experiment to a single cell of interest only. Modeling multi-modal object association functions is required for multi-target trajectory estimations. The tracking module can also share valuable feedback to the following next iteration, assuming applicability. This information can be used to improve segmentation or classification results of the next frame.

The data mining module has the task of selecting and clustering of the so far extracted information in order to concentrate on higher level cell and experimental properties. According to this information the ICP is able to adjust the microscope parameters accordingly.

Our current system setup uses the ProcStarIII board, which is connected by a PCI-X bus to a host computer. In order to increase the scalability especially with respect to banks of microscopes, the system can be extended to use a gigabit Ethernet connection. With this scalability extension the data amount that can be transmitted is by far more limited than with an PCI-X bus and thus data reduction and data mining techniques have to be enforced in real-time. 


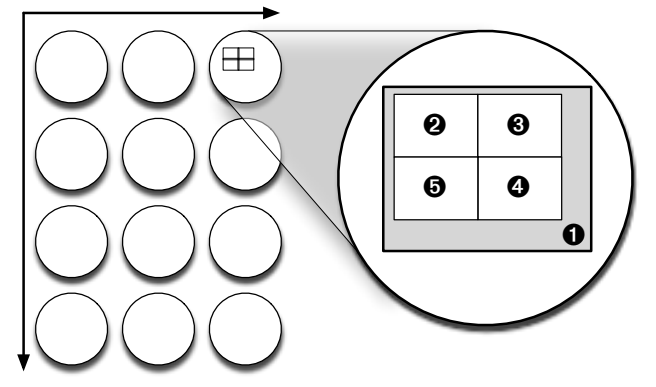

(a) Overview image to determine regions of interest, applied for each well.

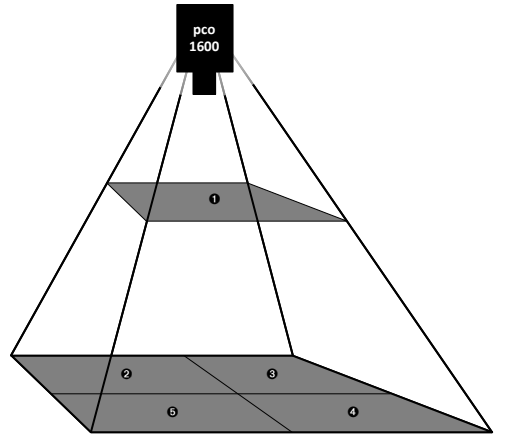

(b) Hierarchy in image capturing, exploiting field of view case-based reasoning.

Figure 4. Typical sizes of multi-well plates range from 6 to more than 1536 wells per plate. The introduction of additional pre-screening steps may be worth the additional effort. In this scenario the pre-screening step decides if four additional detailed images need to be taken. (1 overview image lower magnification factor, 2 - 5 detailed snapshot images at a higher magnification factor.

\section{EXPERIMENTAL RESULTS}

For high-throughput focused systems there are typically time and space constraints that have to be met and high-throughput is related to the amount of biological samples analyzed in a given timeframe, which in turn technically is mainly limited by the amount of handled data and the required processing and transmission time.

The required processing time of a single well without capturing an extra overview image can be approximated by:

$$
\begin{aligned}
\Delta t_{1} & =m_{i}\left(t_{d i}+t_{p}+t_{c}+t_{x}\right) \\
\Delta d_{1} & =m_{i} A
\end{aligned}
$$

where $\Delta t_{1}$ is the processing time for a single iteration (frame) and $m_{i}$ is the number of distinct images taken on detail level $i$ and $t_{d}$ is the average time needed to drive the X-Y-stage to a new position. $t_{p}$ represents the time to take a picture, $t_{x}$ the time for assay-specific image handling, for example capturing a Z-Stack or performing autofocus adjustments and $t_{c}$ specifies the time portion for processing a single image within the embedded visual processing modules. Since the time $t_{x}$ highly depends on the type of processing work, which is highly assay specific, it can typically range from several ms to several minutes.

Estimation of the processing time and data of a single well with capturing an extra overview image:

$$
\begin{aligned}
\Delta t_{2} & =t_{p}+t_{c}+t_{z}+\Delta t_{1}+t_{z} \\
\Delta d_{2} & =A+d_{1}
\end{aligned}
$$

with $t_{z}$ being the time needed for changing the objective. Typical values for the used microscope are: $t_{d i}=0.025 \mathrm{~s}$, $t_{p}=0.1 \mathrm{~s}$ (depending on e.g. the excitation time or the used fluorophores) and $t_{z}=2.0 \mathrm{~s}$. The time $t_{c}$, which specifies the processing and onboard transmission time is dependent on the visual processing modules complexities. The time $t_{x}$ is highly assay-specific and can range between a few microseconds and many minutes depending on the envisaged experiment.

$$
\begin{aligned}
\Delta t_{3} & =t_{p}+t_{c} \\
\Delta d_{2} & =0
\end{aligned}
$$


Investing time for an extra overview image, then the worst case is approximated by $\Delta t_{2}$ and the best case is only $\Delta t_{3}$. Without using an additional overview image the time needed is given by $\Delta t_{1}$. Comparing the amount of data that gets transmitted: without using a overhead image the data is $\Delta d_{1}$, whereas the amount of data using an overview image, which does not get transmitted, is: worst case the same as before $\Delta d_{1}$ and best case $\Delta d_{2}$.

\section{CONCLUSION AND FUTURE WORK}

In this paper we presented a high-throughput sample screening system that enables real-time data analysis and reduction for live cell analysis using fluorescence microscopy. The screening system follows a combined hardwaresoftware architecture for autonomous high-throughput assays, especially targeting experiments for statistical data capturing and evaluation. The usage of an integrated FPGA-DSP combination allows the onboard early signal processing, while at the same time complies with given real-time constraints. Including image pre-processing in the real time loop not only speeds up even complex and time consuming microscope functions, but also allows more complex experiments in high throughput screening. The potential of flexibly configuring the embedded vision module makes it possible to use the system for a variety of different applications where adaptive protocols and/or online data pre-processing and reduction can be helpful or is required. This opens a completely new prospect for high throughput screening and smoothens the way for high content screening on a high throughput scale. Furthermore we proposed a modularized embedded visual processing pipeline scheme that is at the same time flexible with respect to new processing algorithms and has high potential for parallel processing/high throughput.

\section{ACKNOWLEDGMENTS}

The project on which this report is based was sponsored by funds from the Federal Ministry of Economics and Technology according to reference number 16IN0676. The author assumes responsibility for the content of this publication. Sponsored by BMWi on the basis of an enactment with the German Parliament.

\section{REFERENCES}

[1] Lichtman, J. and Conchello, J.-A., "Fluorescence microscopy," Nature Methods 2, 910-919 (Dec 2005).

[2] Axelrod, D., "Total Internal Refection Fluorescence Microscopy in Cell Biology," Traffic 2, 764-774 (2001).

[3] Arndt-Jovin, D., Robert-Nicoud, M., Kaufman, S., and Jovin, T., "Fluorescence Digital Imaging Microscopy in Cell Biology," Science 230(4723), 247-256 (1985).

[4] Guiliano, K., Cheung, W., Curran, D., Day, B., Kassick, A., Lazo, J., Nelson, S., Shin, Y., and Taylor, D., "Systems Cell Biology Knowledge Created from High Content Screening," ASSAY and Drug Development Technologies 3(5), 501-514 (2005).

[5] Wolff, M., Wiedenmann, J., G.U., N., Valler, M., and Heilker, R., "Novel fluorescent proteins for highcontent screening," Drug Discovery Today 11(23-24), 1054-1060 (2006).

[6] Denner, P., Schmalowsky, J., and Prechtl, S., "High-content analysis in preclinical drug discovery," Comb. Chem. High Throughput Screen. 11, 216-230 (Mar 2008).

[7] Gasparri, F., Sola, F., Bandiera, T., Moll, J., and Galvani, A., "High Content Analysis of Kinase Activity in Cells," Combinatorial Chemistry 83 High Throughput Screening 11(7), 523-536 (2008).

[8] K., Z. and S., H., "High content screening - the next challenge: Effective data mining and exploration," Drug Discovery World Winter 2008/09, 27-34 (2008).

[9] Starkuviene, V. and Pepperkok, R., "The potential of high-content high-throughput microscopy in drug discovery," Br. J. Pharmacol. 152, 62-71 (Sep 2007).

[10] Pepperkok, R. and Ellenberg, J., "High-throughput fluorescence microscopy for systems biology," Nat. Rev. Mol. Cell Biol. 7, 690-696 (Sep 2006).

[11] Haney, S. A., LaPan, P., Pan, J., and Zhang, J., "High-content screening moves to the front of the line," Drug Discovery Today 11(19-20), 889 - 894 (2006).

[12] Dragunow, M., "High-content analysis in neuroscience," Nat. Rev. Neurosci. 9, 779-788 (Oct 2008). 
[13] Jamesson, D., Turner, D., J., A., Kennedy, S., Ryan, S., Swainston, N., Griffiths, T., Spiller, D., Oliver, S., White, M. R., Kell, D., and Paton, N., "Information managemant for high content live cell imaging," $B M C$ Bioinformatics 10(226) (2009).

[14] Geisler, T., Ressler, J., Harz, H., Wolf, B., and Uhl, R., "Automated Platform for High-Content and High-Throughput Analytical Screening," IEEE Trans Rob Autom 3, 169-176 (2006).

[15] Seibert, J. A., Boone, J. M., and Lindfors, K. K., "Flat-field correction technique for digital detectors," Medical Imaging 1998: Physics of Medical Imaging 3336(1), 348-354, SPIE (1998).

[16] Lucas J. van Vliet, Frank R. Boddeke, D. S. and Young, I. T., "Image Detectors for Digital Image Microscopy," in [Digital Image Analysis of Microbes: Imaging, Morphometry, Fluorometry and Motility Techniques and Applications], M.H.F. Wilkinson, F. S., ed., 37-64, John Wiley \& Sons, 1 ed. (5 1998).

[17] Hu, M.-K., "Visual pattern recognition by moment invariants," Information Theory, IRE Transactions on $\mathbf{8}$, 179-187 (February 1962).

[18] Khotanzad, A. and Hong, Y., "Invariant image recognition by zernike moments," Pattern Analysis and Machine Intelligence, IEEE Transactions on 12, 489-497 (May 1990).

[19] Brunelli, R., [Template Matching Techniques in Computer Vision: Theory and Practice], Wiley, har/onl ed. (5 2009). 


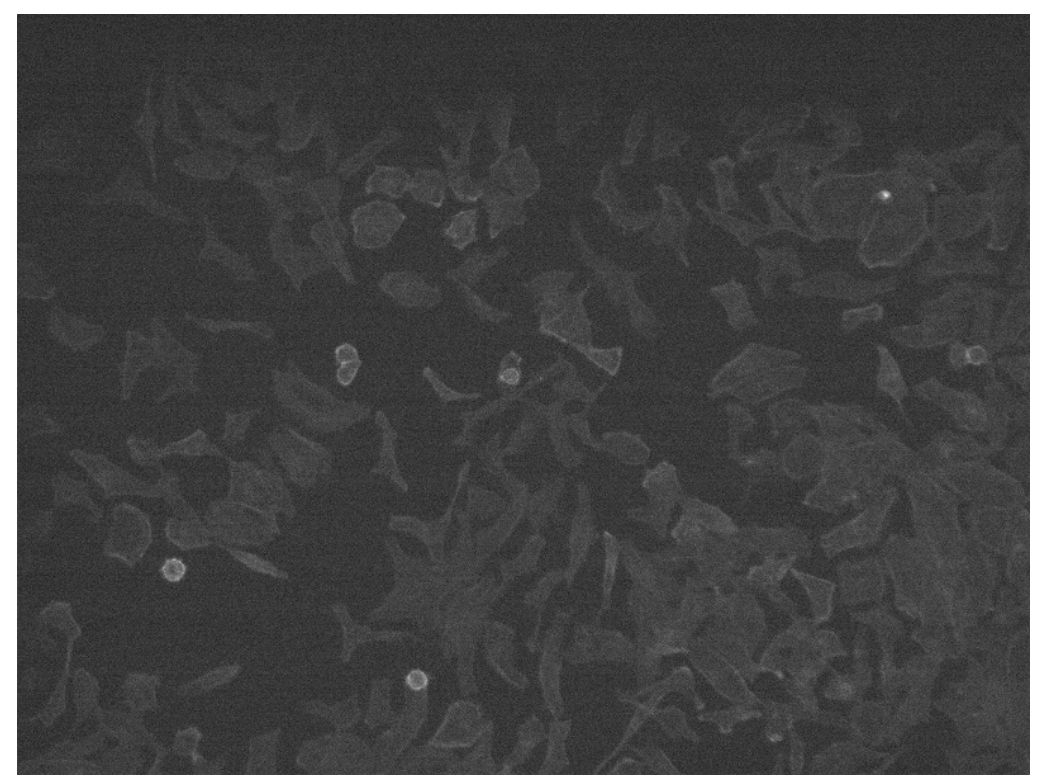

(a) Overview image $10 \times$ objective

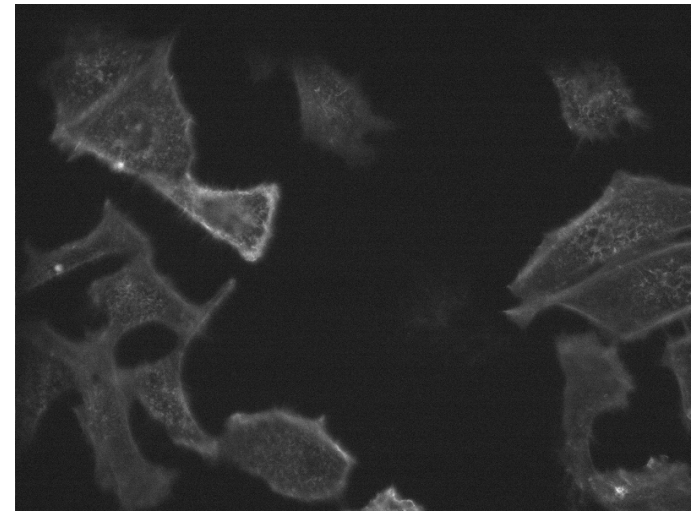

(b) Upper left detail image $40 \times$ objective

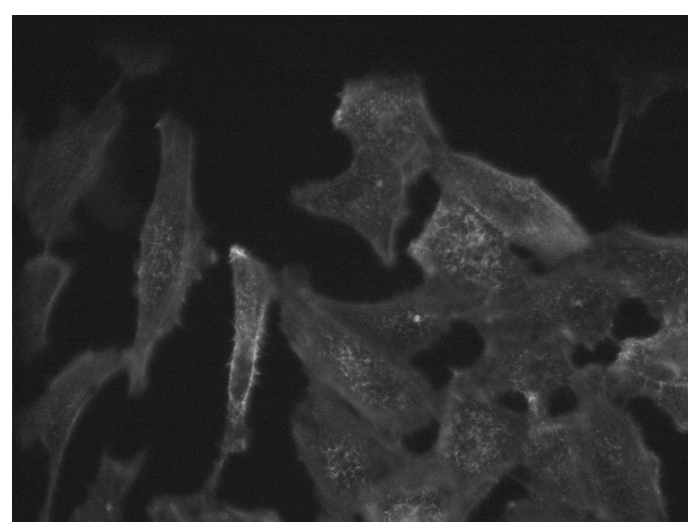

(d) Lower left detail image $40 \times$ objective

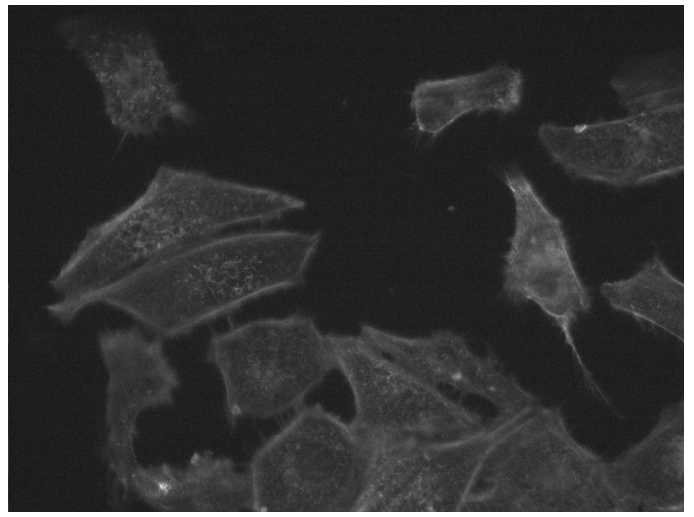

(c) Upper right detail image $40 \times$ objective

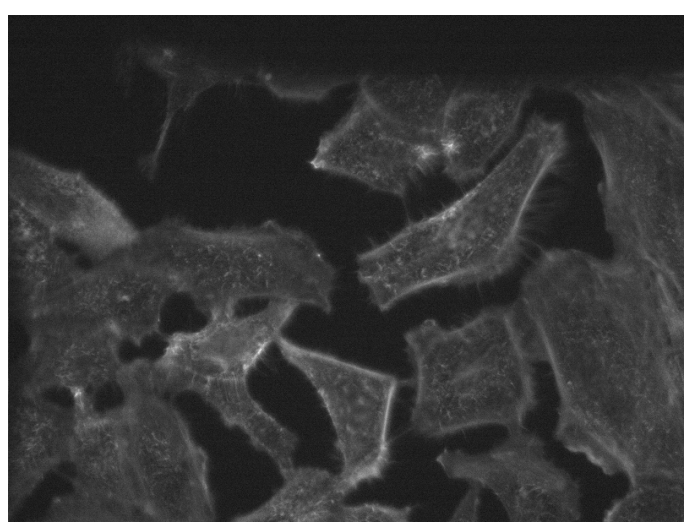

(e) Lower right detail image $40 \times$ objective

Figure 5. Snapshot images captured during a single iteration: In this example 4 detail images have been recorded according to the evaluated overview image. 


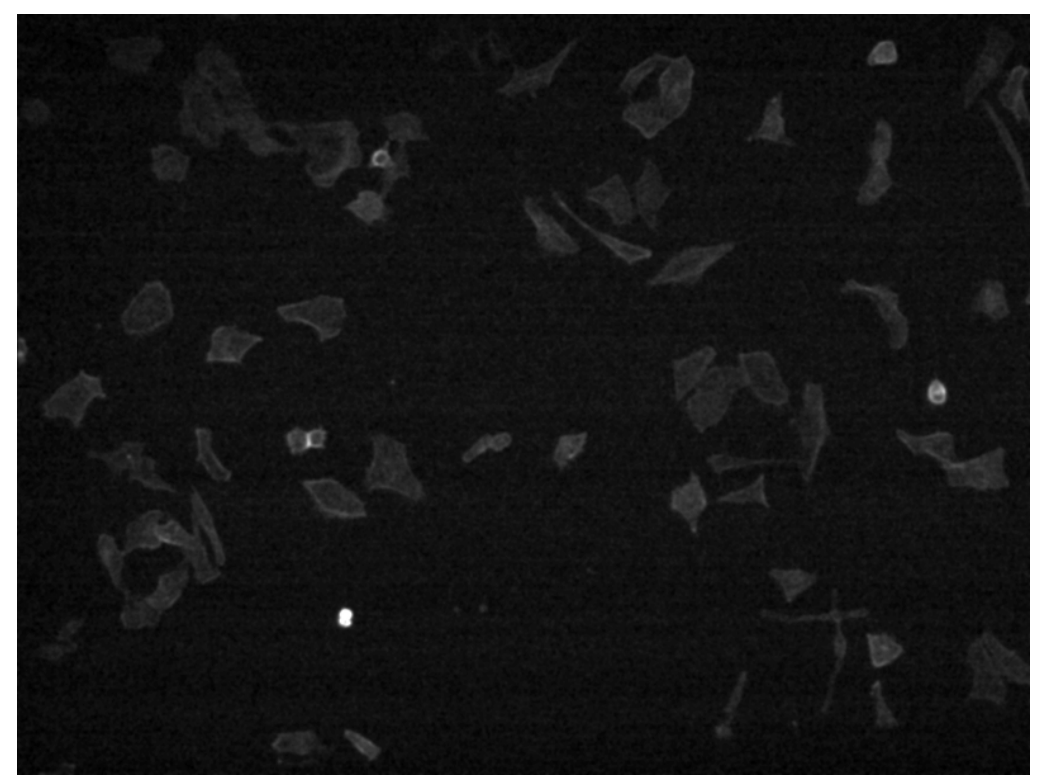

(a) Overview image $10 \times$ objective
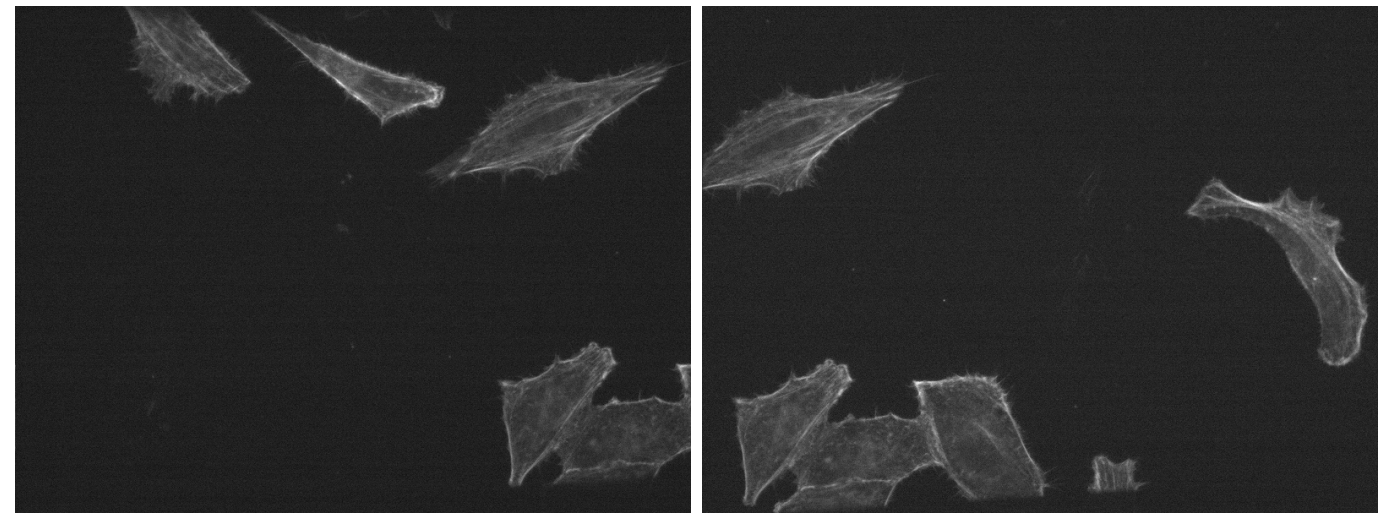

(b) Upper left detail image $40 \times$ objective

(c) Upper right detail image $40 \times$ objective

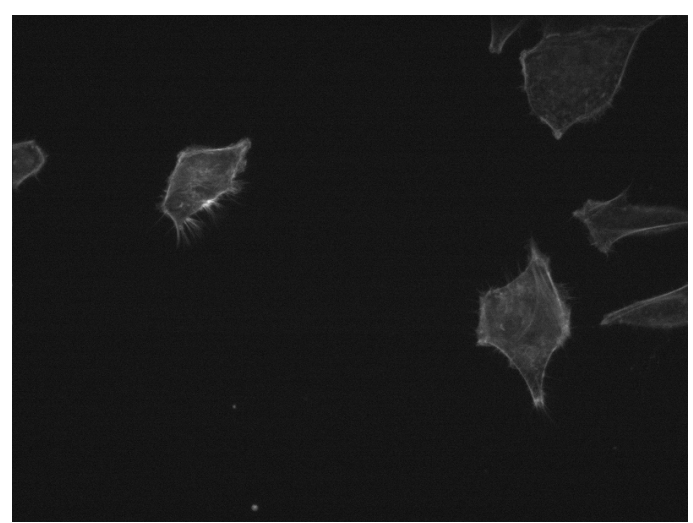

(d) Lower left detail image $40 \times$ objective

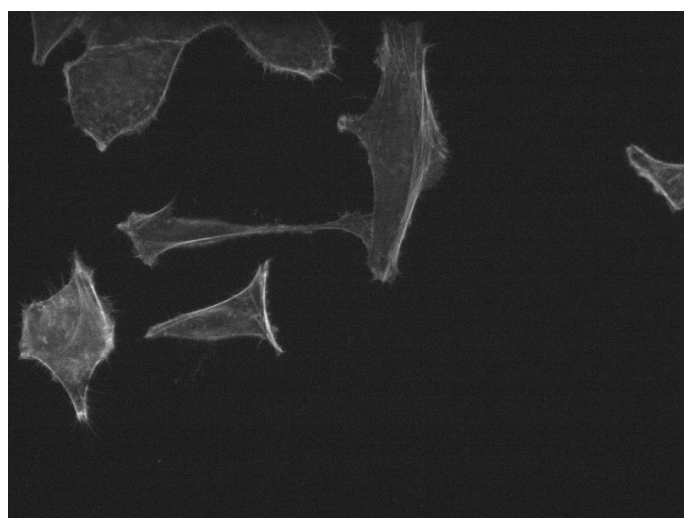

(e) Lower right detail image $40 \times$ objective

Figure 6. Snapshot images captured during a single iteration: In this example 4 detail images have been recorded according to the evaluated overview image. 